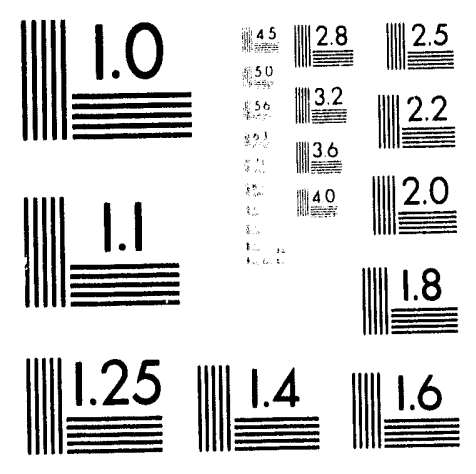



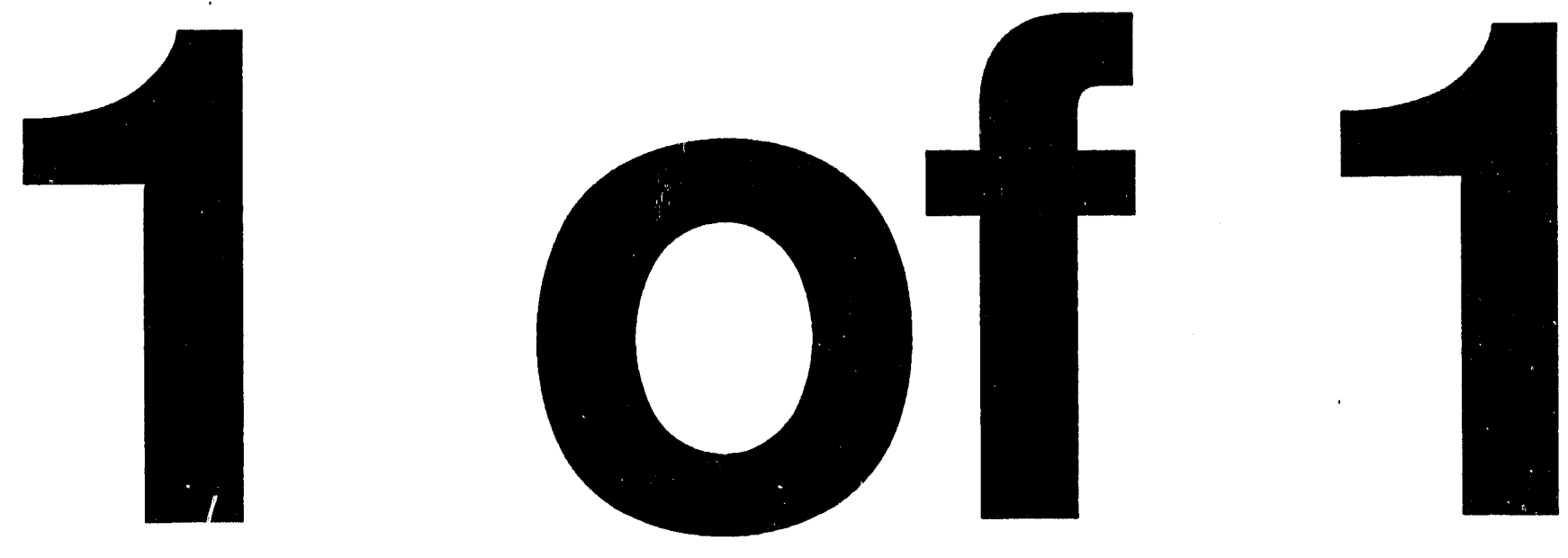


\section{ELECTRON TEMPERATURE GRADIENT DRIVEN INSTABILITY IN THE TOKAMAK BOUNDARY PLASMA}

by

X. Q. Xu, M. N. Rosenbluth, and P. H. Diamond

Memorandum No. UCB/ERL M92/145

15 December 1992

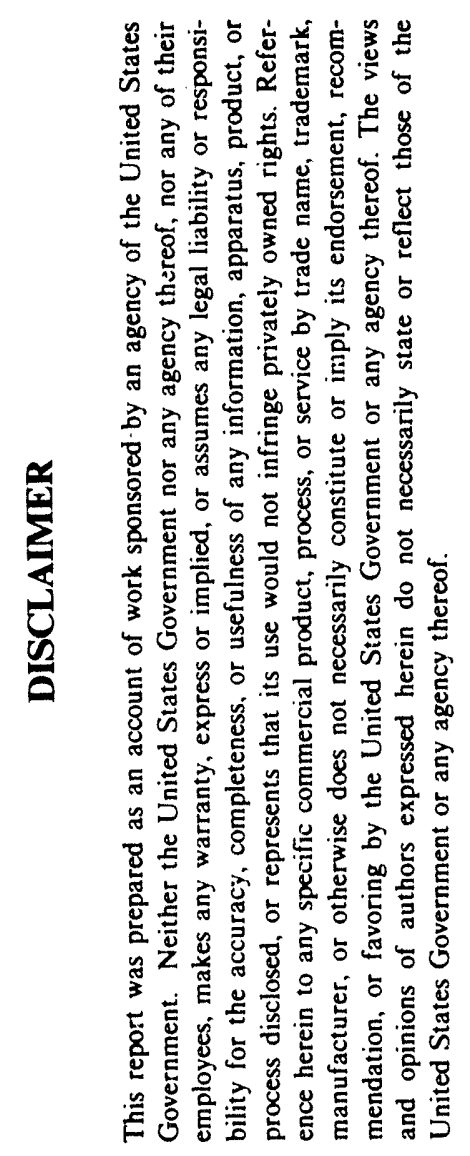

\section{ELECTRONICS RESEARCH LABORATORY}

College of Engineering

University of California, Berkeley

94720 


\title{
Electron Temperature Gradient Driven Instability in the Tokamak Boundary Plasma
}

\author{
X. Q. Xu \\ ERL, U.C.Berkeley \\ and \\ M. N. Rosenbluth and P. H. Diamond \\ Dept. of Phys., U.C. San Diego
}

\begin{abstract}
A general method is developed for calculating boundary plasma fluctuations across a magnetic separatrix in a tokamak with a divertor or a limiter. The slab model, which assumes a periodic plasma in the edge reaching the divertor or limiter plate in the scrape-off layer(SOL), should provide a good estimate, if the radial extent of the fluctuation quantities across the separatrix to the edge is small compared to that given by finite particle banana orbit. The Laplace transform is used for solving the initial value problem. The electron temperature gradient(ETG) driven instability is found to grow like $t^{-\frac{1}{2}} e^{\gamma_{m} t}$.
\end{abstract}

\section{Introduction}

A divertor or limiter is needed in tokamak reactors in the original scenario in order to maintain the low impurity levels required for fusion, and to prevent plasma diffusing across the field lines from reaching the chamber walls. The heat and plasma flow across the separatrix flux surface from the edge to the SOL can be removed by the 
divertor. This is reasonable, since parallel transport is faster than cross-field diffusion. However, electrostatic turbulence has been demonstrated to induce particle transport in boundary plasmas in several tokamak experiments. ${ }^{1,2,3,4}$ The determination of a microturbulent SOL width and the power-handling capability of a divertor or limiter is thus considered to be a major issue for the design of future large tokamaks such as the International Thermonuclear Experimental Reactor (ITER). In recent H-mode experiments, it has been found that boundary plasmas play a very important role in determining the negative electric field, $E_{r}$, reduced edge plasma fluctuations, and improved properties of the main discharge. ${ }^{5}$ Thus, it is important to develop an understanding of the observed plasma f́luctuations and transport across the magnetic separatrix.

Boundary plasma is defined here as the SOL through the last closed flux surface (LCFS) near the magnetic separatrix, and thus includes both open and closed magnetic field lines. The SOL plasma is distinct from the edge plasma because of end-loss to the divertor or limiter plates. Theoretical studies of the turbulent boundary plasma were previously based on assuming either plasmas within closed field line configurations or the SOL plasmas outside the separatrix. The proper consideration of the mode coupling effects between the two regions have become one of most challenging problem in the boundary plasma modeling.

A novel approach to the problem of anomalous transport in the open-field line system starts from the electric interaction between the plasma and the divertor or limiter. ${ }^{6,7,8,9}$ Berk, Ryutov and Tsidulko discovered an electrostatic instability which is driven by the electron temperature gradients in conjunction with end-loss and polarization drift in the gas-dynamic-trap. This idea was generalized to the electromagnetic regime and results have been applied to analyse the electron temperature gradient(ETG) mode in tokamak SOL plasma without magnetic separatrix by Berk, Cohen, Ryutov, Tsidulko, and Xu. ${ }^{10}$ The ETG-modes in the SOL, which resemble the classical rippling modes ${ }^{11,12}$, is primarily electrostatic instability due mainly to elec- 
tron flow along the magnetic field lines under the competing influence of the perturbed potential $\phi(\mathbf{x}, t)=\Phi_{0}(\mathbf{x})+\tilde{\phi}(\mathbf{x}, t)$ and the perturbed temperature $\tilde{T}_{e}$ (analogous to Ohm's law in the case of rippling modes). The unperturbed sheath potential $\Phi_{0}(\mathbf{x})$ exists to equalize the mean electron and ion loss rates. The temperature fluctuations are driven by $\mathbf{E} \times \mathbf{B}_{\mathbf{0}}$ convective relaxation of the equilibrium temperature gradient. In contrast to the case of rippling modes, parillel thermal conduction, $\kappa_{\|} \nabla_{\|}^{2}$, which contributes a strong stabilizing effects to rippling modes, plays a minor role in the ETG-modes in the SOL since the most unstable mode is quasi-flute by nature ( $k_{\|} L<<1$, where $L$ is the axial length of the system). Since the width of the SOL, $\Delta_{x}$, is much smaller than the typical magnetic shear length, $L_{s}\left(\Delta_{x}<<L_{s}\right)$, magnetic shear has no effect on ETG-modes. In this work, we present the principal results that pertain to the electrostatic ETG-mode in the SOL across the magnetic separatrix, using a Laplace transform. Due to the simplicity of the ETG-mode model, it is reasonable to expect that the coupling of many other modes may exhibit features similar to those discussed here. Thus, this method and the results may be of general interest.

\section{Model Equation}

To characterize the region of the boundary plasma which extends across a magnetic separatrix in a tokamak to a limiter or divertor, we consider a slab model. The plasma in a sheared magnetic field $\mathbf{B}(x)=B_{0}\left(\mathbf{e}_{\mathbf{z}}+\frac{x}{L_{0}} \mathbf{e}_{\mathbf{y}}\right)$ is periodic along the axial $z$-direction with period $z=L$ in the plasma edge, and is confined at the ends by two conducting plates in the SOL as shown in Fig. 1. $L_{a}$ is the magnetic shear length. The equilibrium quantities such as $T_{i}, T_{e}, n_{e}$, and $B$ are taken to vary transversely to magnetic field lines in the $x$-direction. In this model, effects on the modes of magnetic trapped particles and magnetic field lines fanning in the SOL on the mode have been omitted.

We now derive the reduced model for the boundary plasmas which includes ionization, radiation and end-loss. The model is derived from the reduced Braginskii fluid 
equations for electrostatic perturbations. ${ }^{13}$ The fluid equations consist of the electron continuity equation, the electron temperature equation, the charge conservation equation and the parallel Ohm's law,

$$
\begin{aligned}
\nabla \cdot \mathbf{j} & =0 \\
\frac{d n_{e}}{d t} & +\nabla_{\|} n_{e} \mathbf{v}_{\|}=S_{n} \\
\frac{3}{2} n \frac{d T_{e}}{d t} & =-\frac{3}{2} n \gamma_{R} T_{e}-\frac{3}{2} n \gamma_{I} T_{e}+\kappa_{\|} \nabla_{\|}^{2} T_{e} \\
\frac{m_{e}}{n_{e} e^{2}} \frac{d j_{\|}}{d t}+\eta_{\| j_{\|}} & =-\nabla_{\|} \phi+\frac{1}{n e} \nabla_{\|} P_{e}+\frac{0.71}{e} \nabla_{\|} T_{e} \\
\mathbf{j}_{\perp} & =-\frac{c \nabla P_{i} \times \mathbf{B}}{B^{2}}-\frac{c^{2} n_{i} m_{i}}{B^{2}} \frac{d \nabla_{\perp} \phi}{d t} \\
\mathbf{v}_{E} & =c \frac{\mathbf{b} \times \nabla \phi}{B} \\
\mathbf{v}_{P_{i}} & =\frac{c \mathbf{b} \times \nabla P_{i}}{e B n_{i}}
\end{aligned}
$$

where $S_{n}$ is the electron ionization rate, $\gamma_{R}$ the radiation loss rate, and $\gamma_{I}$ the electron energy ionization loss rate. In order to focus our attention on the analysis of ETG-mode in the SOL, we keep only the ion $\nabla P_{i}$-drift and ion polarization drift by restricting our analysis to high frequency phenomenon, $\omega \geq \omega_{b e}$, where $\omega_{b e}$ is electron transit frequency. Thus, some modes of interest, e. g., slablike $\eta_{i}$ modes, are not consisdered.

The problem can be solved by the following formal procedure. The vorticity equation (derived from the charge conservation equation) is

$$
-\frac{c^{2} n_{i} m_{i}}{B^{2}} \frac{d}{d t} \nabla^{2} \tilde{\phi}+\nabla_{\| \tilde{j}_{\|}}=0
$$

where the first term represents the parallel component of the vorticity arising from polarization drift. The second term represents field line diffusion (field line bending). The electron end-loss current to the walls and the electron temperature gradient act as destabilizing sources for the electron temperature gradient driven instability. The ion diamagnetism reduces the growth rate at short wavelengths. Substituting the 
generalized parallel Ohm's law

$$
\tilde{j}_{\|}=-\sigma\left(s, k_{y}, k_{\|}\right) \nabla_{\|} \tilde{\phi}
$$

into the vorticity equation, we obtain the eigenmode equation

$$
-\frac{c^{2} n_{i} m_{i}}{B^{2}}\left(s \nabla^{2} \tilde{\Phi}(s)-\nabla^{2} \tilde{\phi}(0)\right)+\sigma\left(s, k_{y}, k_{\|}\right) \nabla_{\|}^{2} \tilde{\phi}=0
$$

where the electron conductivity is

$$
\sigma\left(s, k_{y}, k_{\|}\right)=\frac{\omega_{p e}^{2}}{4 \pi\left(s+i \omega_{E}+\nu_{e i}\right)}\left\{1+\frac{i \omega_{* n_{e}}}{s+i \omega_{E}-\nu_{I}}+\frac{i \alpha \omega_{* T_{e}}}{s+i \omega_{E}-\left(\nu_{R}+\nu_{I}\right)+\frac{2 \kappa \| k_{\| s}^{2}}{3 n_{e}}}\right\}
$$

and we have Laplace transformed the fluid equations with $\phi(x, t) \sim e^{\star t}$ and taken the initial perturbations $T_{e}(0)=n_{e}(0)=0$.

The main complication of the problem comes from the boundary conditions. The eigenmode equation can be solved separately in the edge and the SOL regions based on the corresponding $\tilde{\phi}$ axial boundary conditions along the magnetic field at $z=0$ and $L$. The solutions are then matched at the separatrix $x=0$. The scrape-off layer is divided into two markedly different regions: (1) a narrow sheath region near the conducting wall, with large equilibrium gradients along $z$ of the state variable on the order of the Debye length (to a large extent determined by the electron reflection conditions of the walls, where the electric force dominates actions of the magnetic field), and (2) a large bulk region (in contrast to the sheath) away from the sheaths, with relatively weak equilibrium gradients in $z$. The bulk, in general, is collisional so that fluid equations are appropriate to describe the bulk region plasma flow. The bulk region exhibits many well-known interactions among plasma, waves, neutral gases and impurities. The main thrust of this work to account for fluctuation transport and radial relaxation along $x$ (in addition to classical collisional one) within the axial flow. The sheath, due to its small spatial scale, is treated as boundary conditions here.

\section{Model Sheath Physics}


The solution in the SOL should satisfy the sheath current boundary condition that determines $\phi$ at the sheath edge,

$$
j_{\|}=-\left.\sigma\left(s, k_{y}, k_{\|}\right) \nabla_{\|} \phi\right|_{z=0, L}=j_{\|}^{S h}
$$

where $S h$ denotes the sheath. The expression for the plasma current near the sheath edge is taken to be

$$
j_{\|}^{S h}(L)=-j_{\|}^{S h}(0)=n_{s} e c_{s}\left[1+\gamma_{s i}-\frac{\left(1-\gamma_{s e}\right) v_{T_{e}}}{2 c_{s} \sqrt{\pi}} e^{-\frac{e+(s)}{T_{e}(x)}}\right],
$$

where $c_{s}$ is the sound speed, $v_{T e}$ the electron thermal velocity, and $\gamma_{s}$ the secondary emission coefficient.

The mass difference between electrons and ions results in a large space-charge potential $\Phi_{0}$, and ambipolar flow to the wall demands equal fluxes of negative and positive charge to the walls in equilibrium

$$
J_{\|}^{S h}(0)=J_{\|}^{S h}(L)=0,
$$

which yields the amplitude of the equilibrium potential $\Phi_{0}$ (sheath drop) as

$$
\Lambda \equiv \frac{e \Phi_{0}(x)}{T_{e}(x)}=\frac{1}{2} \ln \left[\frac{m_{i}}{m_{e}} \frac{T_{e}}{T_{i}}\left(\frac{1-\gamma_{e e}}{1+\gamma_{o i}}\right)^{2}\right]
$$

where $\Lambda \simeq 3-4$ except when $\gamma_{s e} \simeq 1$. In the perturbed state, the perturbed current density $j_{\|}$at the sheath edge is related to the perturbation $\tilde{\phi}$ (of the sheath potential $\left.\Phi_{0}\right)$ and the perturbed electron temperature $\tilde{T}_{e}$ by

$$
\tilde{j}_{\|}^{S h}(L)=-\tilde{j}_{\|}^{S h}(0)=n_{e} e c_{s}\left[\frac{e \tilde{\phi}}{T_{e}}-\Lambda \frac{\tilde{T}_{e}}{T_{e}}\right] .
$$

Note that perturbations $\tilde{j}_{\|}^{S h}(L)$ and $\tilde{j}_{\|}^{S h}(0)$ can exist because $\tilde{E}_{y}$ does not have to vanish at the sheath edge though it does vanish at the wall. The first term in Eq. (13) represents an resistive impedance which means prompt removal of all the charges once they pass through the sheath edge. The second term is an inductive impedance due to the temperature fluctuation driven by $\mathbf{E} \times \mathbf{B}_{0}$ convective relaxation of the equilibrium 
temperature gradient, which indicates the finite impedance to the charge flow as it passes through the sheath. It is important to emphasize that there is no net charge accumulated on the plate because the total current to walls has to vanish for an open circuit system. The sheath currents in Eq. (13) set the boundary condition for $\tilde{\phi}$ as Eq. (9).

In the edge, the quantities are periodic in the axial direction, so we can Fourier transform (3), with $\nabla_{\|}$as $i k_{\|}$, which gives

$$
\frac{\partial \tilde{T}_{e}}{\partial t}=-i \omega_{E} \tilde{T}_{c}-i \omega_{\star} T_{e} e \tilde{\phi}-\left(\gamma_{R}+\gamma_{I}\right) \tilde{T}_{e}-\frac{2 \kappa_{\|}}{3 n_{e}} k_{\|}^{2} \tilde{T}_{e}
$$

where

$$
k_{\|}=\frac{2 \pi n}{L}, n=0,1,2, \cdots
$$

In the SOL, the electron temperature equation is the second-order differential equation in $z$ of Eq. (3),

$$
\frac{2 \kappa_{\|}}{3 n_{e}} \frac{\partial^{2} \tilde{T}_{e}}{\partial z^{2}}-\frac{\partial \tilde{T}_{e}}{\partial t}-i \omega_{E} \tilde{T}_{e}-i \omega_{\star} T_{e} e \tilde{\phi}-\left(\gamma_{R}+\gamma_{I}\right) \tilde{T}_{e}=0
$$

and $\tilde{T}_{e}$ should satisfy the sheath heat flux boundary condition. The total sheath heat flux of electrons to the conducting walls sets the boundary condition for axial bulk temperature gradient as

$$
\left.\kappa_{\|} \frac{\partial T_{e}}{\partial z}\right|_{z=0, L}=n_{e} c_{s}\left\{e \phi\left(1-\gamma_{s e}\right)-\frac{T_{e}}{2}-\gamma_{s e} T_{s e}\right\}
$$

where $T_{s e}$ is the temperature of recycled electrons. From Eqs. (17), (9) and (13), we note that, since $\tilde{T}_{e}$ and $\tilde{\phi}$ satisfy different axial boundary conditions at $z=0$ and $L$, we may anticipate that $\tilde{T}_{e}$ and $\tilde{\phi}$ could have different $k_{\| S}$. By neglecting the Debye length scale, axial electron temperature variation is on the order of the equilibrium scale or electron mean free path. Also, since the axial potential variation is on the order of the perturbation scale length, then the axial variation of electron temperature $\tilde{T}_{e}$ is much more rapid than that of potential $\tilde{\phi}$ at the sheath edge, for the most unstable mode. The boundary layer problem is now well defined. Within the boundary layer 
$z \in(L-\epsilon, L)$ with $\epsilon<<L, \tilde{T}_{e}$ is not proportional to $\tilde{\phi}$, and the sheath heat flux boundary condition on the electron flow to the wall forces $\kappa_{\|} \nabla_{\|} \tilde{T}_{e}$ to satisfy the boundary condition. But within the axial bulk region $z \in(\epsilon, L-\epsilon)$, we assume that $\tilde{T}_{e}$ is proportional to $\tilde{\phi}$. For $\tilde{\phi}$, we may assume that $\left.\left.\nabla_{\|} \tilde{\phi}\right|_{L-\epsilon} \simeq \nabla_{\|} \tilde{\phi}\right|_{L}+O(\epsilon)$, and that other quantities have similar behavior. Thus, to order $\epsilon$, we may assume our sheath boundary condition at $L-\epsilon$. The solution obtained by employing this boundary condition gives correct expressions for the elertron current into the sheath within $\epsilon$ and therefore correct expressions for instability away from the edge of the boundary layer. However, the solution cannot be expected to provide a proper description for the temperature near the sheath. For radiative instability in a divertor plasma, the sheath heat flux boundary condition becomes important in determining the axial equilibrium electron temperature profile, and the argument given above is invalid. ${ }^{14}$

\section{Eigenmode Equation}

Our approach is through the initial-value problem. The motivation is that when the usual normal-mode method yields no discrete unstable solution, we will find that there is a continuum of unstable mode. This results in an initial perturbation growing as a product of exponential and a negative fractional power of time. ${ }^{15,16}$

The complete set of equations and boundary condition, after Laplace transform with parameter $s$, are

$$
\begin{aligned}
\rho_{s}^{2} \nabla_{\perp}^{2} \Phi_{E}(s)-k_{\|}^{2} A_{E}^{2} \Phi(s) & =\Psi_{E}(s, x, y, z), x<0 \\
\rho_{s}^{2} \nabla_{\perp}^{2} \Phi_{S}(s)+A_{S}^{2} \nabla_{\|}^{2} \tilde{\Phi}(s) & =\Psi_{S}(s, x, y, z), x>0 \\
\left.\nabla_{\|} \Phi_{S}(s)\right|_{z=0, L} & = \pm B_{S} \Phi_{S}(s), x>0
\end{aligned}
$$

where $\Phi(s)$ is periodic in $z$ for all $x$. Also, by neglecting small effects of radiation $\gamma_{R}$ and ionization $\gamma_{I}$, we have

$$
\begin{aligned}
& \dot{A}_{S}^{2}=\frac{v_{T e}^{2}}{\left(s+i \omega_{E}-i \omega_{* p_{i}}\right)\left(s+i \omega_{E}+\nu_{e i}\right)}\left[1+\frac{i \omega_{* n_{e}}}{s+i \omega_{E}}+\frac{i \alpha \omega_{* T_{e}}}{s+i \omega_{E}+\frac{2 \kappa_{\|}}{3 n_{e}} k_{\| S}^{2}}\right] \\
& A_{E}^{2}=\frac{v_{T e}^{2}}{\left(s+i \omega_{E}-i \omega_{m p_{i}}\right)\left(s+i \omega_{E}+\nu_{e i}\right)}\left[1+\frac{i \omega_{* n_{e}}}{s+i \omega_{E}}+\frac{i \alpha \omega_{* T_{e}}}{s+i \omega_{E}+\frac{2 \kappa_{\|} \|}{3 n_{e}} k_{\|}^{2}}\right],
\end{aligned}
$$




$$
\begin{gathered}
B_{S}=-\frac{\left(s+i \omega_{E}+\nu_{e i}\right) \nu_{L}}{2 \omega_{b e}^{2} L} \frac{s+i \omega_{E}+i \Lambda \omega_{*} T_{e}+\frac{2 \kappa_{\|} k_{\| s}^{2}}{3 n_{e}}}{\left(s+i \omega_{E}+\frac{2 \kappa_{\|} k_{\| s}^{2}}{3 n_{e}}\right)\left(1+\frac{i \omega_{* n_{e}}}{s+i \omega_{E}}\right)+i \alpha \omega_{*} T_{e}}, \\
\Psi_{E}\left(s, \quad x, k_{y}, z\right)=\Psi_{S}\left(s, x, k_{y}, z\right)=\frac{\rho_{s}^{2} \nabla_{\perp}^{2} \phi(0)}{s+i \omega_{E}-i \omega_{* p_{i}}},
\end{gathered}
$$

where $\omega_{b e}=v_{T_{e}} / L$ is electron axial bounce frequency and $\nu_{L}=2 c_{s} / L$ equilibrium ion axial loss rate( $v$ here the factor of 2 comes from the contribution from two ends), all subscripts $E$ represent the edge quantities and $S$ the SOL quantities. We have taken initial perturbations $n_{e}(0)=0$ and $T_{e}(0)=0$, and $k_{\|}=\frac{2 \pi n}{L}+\frac{k_{y} x}{L_{0}}(n=0,1,2, \cdots)$. The parallel wavenumbers in the SOL $k_{\| S}$ are determined by the sheath boundary condition Eq. (20). Notice that Eq. (20) is obtained in the case of the magnetic field normal to the walls. In a sheared slab where the magnetic field is mainly in the $z$ direction and hits the wall (limiter or divertor plate) at small angle, the bulk plasma flow in the SOL is essentially directed along the magnetic field. But the total electric potential drop between plasma and wall has been shown by Chodura ${ }^{17}$ to be normal (axial), and is fairly insensitive to the magnitude and angle of the magnetic field. Thus, as a good approximation, Eq. (20) is still valid in a sheared slab by replacing $\nabla_{\|} \Phi_{S}(s)$ with $\frac{d \Phi_{S}(\bullet)}{d z}$.

For simplicity, we assume that

$$
\Psi_{E}\left(s, x, k_{y}, z\right)=\Psi_{S}\left(s, x, k_{y}, z\right)=\frac{\rho_{s}^{2} \phi(0)}{s+i \omega_{E}-i \omega_{* p_{i}}} \delta(x),
$$

which means that we introduce an initial charge perturbation at the separatrix. It can be proven that the final time asymptotic behavior is insensitive to the location of initial perturbations. However, the mode structures will be modulated by the initial charge perturbation profile.

Integrating eigenmode equations (18) and (19) across the separatrix at $x=0$, we obtain the matching condition

$$
\left.\frac{\partial \Phi_{E}(s)}{\partial x}\right|_{x=0}-\left.\frac{\partial \Phi_{S}(s)}{\partial x}\right|_{x=0}=\frac{\phi(0)}{s+i \omega_{E}-i \omega_{* p_{i}}}
$$

and the condition

$$
\Phi_{E}\left(s, x=0, k_{y}, z\right)=\Phi_{S}\left(s, x=0, k_{y}, z\right) .
$$




\section{Mechanism of E'TG-Mode in the SOL}

In the region $x>0$, the solutions for $\Phi_{S}(s)$ are the usual Berk et al.' $\mathrm{s}^{8}$ mode when the electron temperature gradient and end-loss are present in the SOL for a shearless slab. Thus we have

$$
\begin{aligned}
\Phi_{S}(s) & =\sum_{l} C_{l}^{S} \cos \left[k_{\| S, l}(z-L / 2)\right] e^{i k_{x, l}(a) x}, \\
\rho_{a}^{2} k_{x, l}^{2}(s) & =-A_{a}^{2} k_{\| S, l}^{2}-k_{y}^{2} \rho_{s}^{2},
\end{aligned}
$$

where we assume that the outer radial boundary is at infinity $\left(L_{x} \rightarrow \infty\right)$, therefore only the transmitted wave through the separatrix in the SOL is kept. In the Appendix, a finite radial length system with a reflected wave in the SOL is discussed.

From the sheath boundary condition $\left.\nabla_{\|} \Phi(s)\right|_{z=0, L}= \pm B_{S} \Phi(s)$, we obtain

$$
\left(s+i \omega_{E}-i \omega_{* p_{i}}\right) k_{\perp}^{2} \rho_{e}^{2} \frac{\tan \left(\frac{k_{\| S, L} L}{2}\right)}{k_{\| S, l} L}=-\nu_{L} \frac{s+i \omega_{E}+i \Lambda \omega_{* T_{e}}+\frac{2 \kappa_{\|} k_{\| S, l}^{2}}{3 n_{e}}}{\left(s+i \omega_{E}+\frac{2 \kappa_{\|} k_{\| S, l}^{2}}{3 n_{e}}\right)} .
$$

Note that $k_{x, l}(s)$ is not a Fourier transform quantity in $x$. The functional relation between $k_{x, l}(s)$ and $s$ is given by Eqs. (29) and (30) (where the function may be many-valued, labeled by $l$ ). We suppose that for some finite range of real values of $s$, the corresponding values of one branch of $k_{x, l}(s)$ are complex.

For $\left(k_{\| S, 0} L / 2\right)<<1$, hence $\frac{2 \kappa_{\|} k_{\| e, 0}^{2}}{3 n_{e}}<s$, we have

$$
\frac{1}{2}\left(s+i \omega_{E}-i \omega_{* p_{i}}\right) k_{\perp}^{2} \rho_{s}^{2}=-\nu_{L} \frac{s+i \omega_{E}+i \Lambda \omega_{*} T_{e}}{\left(s+i \omega_{E}\right)}
$$

where $k_{\perp}^{2}=k_{y}^{2}+k_{x, 0}^{2}(s)$. The square root in Eq. (31) for $k_{x, 0}(s)$ must be rendered definite by branch cuts in the complex $s$-plane radiating outwards from the zeros of $k_{x, 0}^{2} ;$ the path of integration in the inversion of the Laplace transform must not cross these cuts. Strictly speaking, the case $k_{\| S, l}=0$ is now forbidden.

Note that in a system without a magnetic separatrix, $k_{x, 0}(s)$ becomes a Fourier transform in $x$ (that is, a real wavenumber $k_{x}$ ), and Eq. (31) is the dispersion relation of the electron temperature gradient driven instability given by Berk, Ryutov, and 
Tsidulko. ${ }^{8}$ The physical picture of this instability is as follows. For a fluid discription, electron collisions have been assumed to be sufficient to maintain a Maxwellian distribution and to populate the velocity-space loss cone. In equilibrium, the total current density due to electrons and ions impinging on the plate is zero, setting the equilibrium sheath potential $\Phi_{0}$ as indicated in Eq. (12). In this picture, fast electrons with velocity $v>\sqrt{\frac{2 e \Phi_{0}}{m_{e}}}$ are lost to the walls and slow electrons with $v<\sqrt{\frac{2 e \Phi_{0}}{m_{e}}}$ will be reflected by the sheath potential $\Phi_{0}$. Since we assume an equilibrium electron temperature in the radial " $x$ "-direction, and consider a seed potential $\tilde{\phi}$ in the form of a periodic variation in the $y$-direction, the mean electron energy fluctuations are driven by $E_{y} \times B_{0}$ convective relaxation of the equilibrium temperature gradient. Some electrons will gain enough energy to cross the sheath potential hill and become lost to the wall, and some electrons will lose energy and become confined, either of which in turn generates the local perturbed parallel current. From charge conservation, the perturbed ion polarization current will be generated to cancel the divergence of the parallel current. The phase shift of the two current fluctuations results in amplification of the seed potential $\tilde{\phi}$, thus causing the instability. However, because of the periodic nature of perturbations in the $y$-direction, total current to the walls vanishes after integrating the local perturbed parallel current density $\tilde{j}_{\|}$over $y$. Thus, there is no net charge accumulation on the walls. In comparison, rippling modes result from the coupling of electric resistivity fluctuations to potential and current fluctuations through Ohm's law. The electric resistivity fluctuations are driven by $\mathbf{E} \times \mathbf{B}_{\mathbf{0}}$ convective relaxation of the average temperature gradient. By replacing $\nu_{L}$ with $k_{\|} u$ where $u$ is the directed electron velocity, $u=-\frac{J_{\varrho}}{n_{\mathrm{e}} e}$ and $J_{0}$ is the parallel equilibrium current, a similar dispersion relation to $\mathrm{Eq}$. (31) has been obtained for rippling modes (or current-convective instability) by Kadomtsev and Pogutse. ${ }^{11,12}$ Thus, for a historical reason and better description, we may also call the ETG-mode in the SOL to be the sheath driven rippling modes. Since the parallel equilibrium current $J_{0}$ is typically some modest fraction of the ion current $n_{e} e c_{s}$ (that is, $J_{0} \sim \delta_{s} n_{e} e c_{s}$ 
with $\delta_{s}<1$ ), the growth rate of the rippling modes is normally smaller by a factor of $\sqrt{\frac{\Lambda}{\delta_{s}}}$ and the large pa-nllel heat conductivity contributes a strongly stabilizing effect on the rippling modes for finite $k_{\|}$. A quantitive comparison with rippling modes is possible and is deferred to future publication.

For $k_{\| S, l} L / 2 \simeq l \pi, l=1,2,3, \cdots$, we can solve Eq. (30) perturbatively and find that $s_{I} \simeq l \pi \frac{\omega_{b c}}{k_{\perp} \rho_{c}}$ with a small real part $s_{R} \simeq-\frac{\nu_{L}}{k_{\perp}^{2} \rho_{c}^{2}}\left\{1-\frac{\Lambda \omega_{*} r_{c}}{\theta_{I}}\right\}$. In this case, electrons travel with the waves in the axial direction and the ohmic resistance of the bulk plasma has been ignored. The first term in $s_{R}$ comes from potential perturbation $\tilde{\phi}$ and the second term from electron temperature perturbation $\tilde{T}_{e}$ due to $\mathbf{E} \times \mathbf{B}_{0}$ convection of the equilibrium temperature gradient. Since electron temperature perturbation $\tilde{T}_{e}$ is in phase with potential perturbation $\tilde{\phi}$, and $\frac{e \bar{\phi}}{T_{e}}$ dominates over $\frac{\tilde{T}_{e}}{T_{e}}$ in the sheath current perturbation $\tilde{j}_{\|}^{S h}$ for $s_{I}>>\Lambda \omega_{*} T_{e}$ (as shown in Eq. (13)), harmonics of $l>0$ modes are damped by the sheath resistive impedance.

It is natural to ask whether magnetic shear affects the ETG-mode in the SOL. A similar issue has been addressed in a classic paper by Roberts and Taylor. ${ }^{18}$ They analysed the effects of the magnetic shear on the gravitational resistive instability in an open-field line system (e. g. , stellarator) and discovered that the convective cells twisted to conform to the field lines. In a system with weak shear, such as the SOL of a tokamak, the twisting of the ETG-mode by the sheared magnetic field can be estimated by comparing the two terms in $k_{\|}=k_{z}+\frac{k_{y} x}{L_{0}}$. We find that $\epsilon \equiv$ $\frac{k_{y} x}{k_{z} L_{0}} \sim \frac{\Delta_{x} \omega_{b c}^{2}}{L_{0} s^{2}}$ where $\Delta_{x}$ is the width of the SOL and $k_{z}$ has been approximately obtained from Eq. (29). For a typical DIII-D tokamak boundary plasma parameters, $\Delta_{x} \sim 1 \mathrm{~cm}, L_{s} \sim 1 \mathrm{~m}$, and $s \geq 3 \omega_{\text {be }}, \epsilon$ is typically of order $10^{-3}$. This means that for the weakly sheared system like the SOL of tokamak, the influence of magnetic shear on ETG-mode can be ignored.

\section{Potential Fluctuation across a Separatrix}

Considering the periodic nature of the structure in $z$ inside the separatrix, and the continuity of $E_{z}$ at the separatrix, we can expand the outside solution $\Phi_{S, l}(s)=$ 
$\cos \left[k_{\| S, l}(z-L / 2)\right] e^{i k_{x, l}(s) x}$ as a cosine series for the inside solution $\Phi_{E}(s)$

$$
\Phi_{E}(s)=\sum_{n} C_{n}^{E} \cos \left[\frac{2 \pi n}{L}(z-L / 2)\right] f_{n}(x) .
$$

From eigenmode equation (18), $f_{n}(x)$ is determined by

$$
\rho_{s}^{2} \frac{d^{2} f_{n}}{d x^{2}}-\left\{k_{y}^{2} \rho_{s}^{2}+\left(\frac{2 \pi n}{L}+\frac{k_{y} x}{L_{s}}\right)^{2} A_{E}^{2}\right\} f_{n}=0 .
$$

From the matching condition of the radial derivative $\left.\frac{\partial \Phi(s)}{\partial x}\right|_{x=0}$ and $\left.\Phi(s)\right|_{x=0}$ at the separatrix, we have

$$
\begin{aligned}
& C_{0}^{E}=\sum_{l} \frac{2 C_{l}^{S} \sin \left(k_{\| S, l} L / 2\right)}{k_{\| S, l} L}, \\
& C_{n}^{E}=\sum_{l} \frac{4 C_{l}^{S}(-1)^{n} k_{\| S, l} \sin \left(k_{\| S, l} L / 2\right)}{\left[k_{\| S, l}^{2}-\left(\frac{2 \pi n}{L}\right)^{2}\right] L} \\
& \sum_{l} \frac{2 C_{l}^{S}}{L} \frac{(-1)^{n} k_{\| S, l} \sin \left(k_{\| S, l} L / 2\right)}{\left[k_{\| S, l}^{2}-\left(\frac{2 \pi n}{L}\right)^{2}\right]}\left(\alpha_{n}-i k_{x, l}\right)=\frac{\phi(0) L \delta_{n 0}}{s+i \omega_{E}-i \omega_{* p_{i}}},
\end{aligned}
$$

where we have set $f_{n}(0)=1$ and $\alpha_{n}=\frac{d f_{n}(0)}{d x}$. Inside the separatrix $x \simeq 0$, we have approximately $f_{n}(x) \sim e^{\alpha_{n} x}$. Using the solutions in Eq. (31) for $k_{\| S, 0} L / 2$ and $k_{\| S, l} L / 2 \simeq l \pi, l=1,2,3, \cdots$, we have

$$
\begin{aligned}
C_{0}^{S}\left(\alpha_{0}(s)-i k_{x, l}(s)\right) & =\frac{\phi(0) L \delta_{n 0}}{s+i \omega_{E}-i \omega_{* p_{i}}}, \\
C_{n}^{S}\left(\alpha_{n}(s)-i k_{x, n}(s)\right) & =0 . \quad n=1,2,3, \cdots
\end{aligned}
$$

Because the physical solution requires $\operatorname{Re}\left(\alpha_{n}\right)>0$ and $\operatorname{Im}\left(k_{x, l}\right)>0$, the above matrix equation has no solution when $\phi(0)=0$, except for drift waves with frequencies $s=i \omega_{* P_{i}}-i \omega_{E} ;$ thus, there is no normal mode for $\tilde{E}_{x} \neq 0$. With $\alpha_{n}(s)-i k_{x, n}(s) \neq 0$, we have $C_{n}^{S}=0$ for $n \neq 0$ and

$$
C_{0}^{E}=C_{0}^{S}=\frac{\phi(0)}{\left(\alpha_{0}(s)-i k_{x, 0}(s)\right)\left(s+i \omega_{E}-i \omega_{* p_{i}}\right)} .
$$

The Laplace inversion theorem,

$$
\begin{aligned}
\phi_{S}(\mathbf{x}, t) & =\frac{1}{2 \pi i} \int_{c} d s \sum_{l} C_{l}^{S}\left(k_{x, l}(s), k_{y}\right) \\
& \times \cos \left[k_{\| S, l}\left(z-\frac{L}{2}\right)\right] \exp \left\{i\left[k_{x, l}(s) x+k_{y} y\right]+s t\right\}
\end{aligned}
$$


is used to invert the Laplace transform, where the contour $c$ is within the half plane of analyticity of $\Phi_{S}(s)$ and runs parallel to the imaginary axis.

The numerical solution of Eq. (31) with $\omega_{* P_{i}}=0$ is plotted in Fig. 2 in the complex $s$-plane. The solid curve represents the case with parameter $k_{x, 0}=0$ and $\hat{k}$ variable, and the dashed curve with parameter $\hat{k}=1.8$ and $k_{x, 0}$ variable, where $\hat{s}=\left(s+i \omega_{E}\right) \nu^{-\frac{1}{3}}, \hat{k}=k_{y} \rho_{s} \nu^{-\frac{1}{3}} . \nu=\frac{\nu_{L} k_{\nu} \rho_{e}}{\Lambda \omega_{v} T_{e}}$. We find that the maximum growth rate is located at

$$
\begin{aligned}
\frac{k_{\| S, 0} L}{2} & <1, \\
k_{x, 0}(s)= & 0, \\
k_{y m} \rho_{s}= & 1.8\left(\frac{L_{T_{e}} \nu_{L}}{\Lambda c_{s}}\right)^{\frac{1}{3}}, \\
s_{m}= & \gamma_{m}-i \omega_{m}, \\
\gamma_{m}= & 0.38\left[\left(\frac{\Lambda c_{s}}{L_{T_{e}}}\right)^{2} \nu_{L}\right]^{\frac{1}{3}}, \\
\omega_{m}= & 0.52\left[\left(\frac{\Lambda c_{s}}{L_{T_{e}}}\right)^{2} \nu_{L}\right]^{\frac{1}{3}} .
\end{aligned}
$$

It has been shown that the ion diamagnetism stabilizes the mode at short wavelength $(\hat{k} \sim 10)$, but has little effect on the maximum growth rate. ${ }^{8,10}$ The branch cut is given by the dashed curve in Fig. 2. To find the asymptotic behavior of the integrals $\Phi_{\mathcal{S}}(\mathbf{x}, t)$, we deform the contour $c$ to the left to circle the branch cut. On the basis that the most unstable mode and its neighbouring modes dominate the others after some time of exponential growth, the "Gaussian" approximation to the integral is obtained by truncating the argument of the exponent in the above equation to second degree in $k_{x, 0}^{2}$, changing integral variable from $s$ to $k_{x, 0}^{2}$ and integrating the resulting expression in closed form along the real $k_{x, 0}^{2}$ axis. ${ }^{19}$

Thus, asymptotic behavior of the potential fluctuation $\phi_{S}(\mathbf{x}, t)$ can be found as

$$
\phi_{S}(\mathbf{x}, t) \sim t^{-\frac{1}{2}} \exp \left\{\left(\gamma_{m}-i \omega_{m}\right) t+i k_{y m} y-\frac{(1-i)\left(k_{y m} x\right)^{2}}{\gamma_{m} t}\right\}
$$

Thus, if the initially introduced potential perturbation is finite, the amplitude of the wavepacket grows like $t^{-\frac{1}{2}} e^{\gamma_{m} t}$, where $t^{-\frac{1}{2}}$ arises from interference of components 
of the packet due to the field matching conditions Eqs. (26) and (27) at the separatrix $x=0$. We also see that the amplitude of the wavepacket depends only weakly on $x$.

To investigate the asymptotic behavior of edge potential fluctuation $\phi_{E}(\mathbf{x}, t)$, Eq. (33) has to be solved. We consider two cases:

Case 1. Shearless slab model when $L_{\star} \rightarrow \infty$. Eq. (33) gives $f_{n}(x)=e^{\alpha_{n}(s) x}$ with

$$
\rho_{s}^{2} \alpha_{n}^{2}(s)=A_{E}^{2}\left(\frac{2 \pi n}{L}\right)^{2}+k_{y}^{2} \rho_{s}^{2}
$$

The asymptotic evaluation proceeds as in the case of $\phi_{S}(\mathbf{x}, t)$, and we obtain the potential fluctuation inside the separatrix as

$$
\phi_{E}(\mathbf{x}, t) \sim t^{-\frac{1}{2}} \exp \left\{\left(\gamma_{m}-i \omega_{m}\right) t+k_{y m} x+i k_{y m} y\right\}
$$

Comparing $\phi_{E}(\mathbf{x}, t)$ with $\phi_{S}(\mathbf{x}, t)$, we found that $\phi_{E}(\mathbf{x}, t)$ has the same growth rate, but decays slowly in $x$ inside the separatrix, on the order of $k_{y m}^{-1}$ for $k_{y m}>0$.

Case 2. Sheared slab model. Eq. (33) can be rewritten as

$$
\frac{d^{2} f_{n}}{d \xi_{n}^{2}}-\left(\lambda+\frac{\xi_{n}^{2}}{4}\right) f_{n}=0
$$

with $\xi_{n}=\sqrt{\frac{2 A_{E}}{L_{0} k_{y} \rho_{\varepsilon}}}\left(k_{y} x+\frac{2 \pi n L_{0}}{L}\right)$ and $\lambda=\frac{k_{y} \rho_{0} L_{0}}{2 A_{E}}$. Eq. (44) then is a parabolic cylinder equation with even solution given as Whittaker's function, ${ }^{20}$

$$
f_{n}\left(\xi_{n}\right)=\frac{U\left(\lambda, \xi_{n}\right)}{U\left[\lambda, \xi_{n}(x=0)\right]}
$$

It may be conjectured that magnetic shear plays a significant role on this mode, since in contrast to the SOL there exists a corresponding flute mode with toroidal mode number $\mathbf{n}=0$ in the edge. For $n=0$ mode near the magnetic separatrix $x \simeq 0, \lambda>0$ and $\lambda>>\xi_{0}^{2}$, we have

$$
U\left(\lambda, \xi_{0}\right)=\frac{\sqrt{\pi}}{2^{\frac{\lambda}{2}+\frac{1}{2}} \Gamma\left(\frac{3}{4}+\frac{\lambda}{2}\right)} e^{\sqrt{\lambda} \xi_{0}}
$$

Therefore

$$
f_{0}(x)=e^{k_{y} x}
$$


which yields $\alpha_{0}=k_{y}>0$. This is a reasonable result since near a separatrix $x \simeq 0$, the magnetic shear term plays a minor role. For most interesting regions, we can expand $U\left(\lambda, \xi_{0}\right)$ for $\xi_{0}$ large and $\lambda$ moderate. When $\xi_{0}>|\lambda|$, we have

$$
U\left(\lambda, \xi_{0}\right) \sim \xi_{0}^{-\lambda-\frac{1}{2}} e^{-\frac{\xi_{0}^{2}}{4}}
$$

For $\lambda^{\circ}<<1$,

$$
f_{0}(x) \simeq\left\{k_{y} x \sqrt{\frac{2 L}{k_{y} \rho_{s} L_{a}} \frac{\omega_{b e}}{s_{m}}}\right\}^{-\frac{1}{2}} e^{-\frac{1}{2} \frac{k_{y} \rho_{a} L}{L_{0}} \frac{\omega_{b e}}{s_{m}}\left\{\frac{s}{\rho_{0}}\right\}^{2}} .
$$

The asymptotic evaluation proceeds as in Case 1 , we find asymptotically that

$$
\begin{aligned}
& \phi_{E}(\mathbf{x}, t) \simeq\left\{\left|k_{y} x \sqrt{\frac{2 L}{k_{y} \rho_{\theta} L_{b}} \frac{\omega_{b e}}{s_{m}}}\right|\right\}^{-\frac{1}{2}} e^{-\frac{1}{2} \frac{L_{y} \rho_{0}}{L_{\theta}} \frac{\omega_{b e}}{b_{m}}\left\{\frac{2}{\rho_{\theta}}\right\}^{2}} \\
& \times t^{-\frac{1}{2}} \exp \left\{\left(\gamma_{m}-i \omega_{m}\right) t+i k_{y m} y\right\} \text {. }
\end{aligned}
$$

Since the time asymptotic behavior of the potential fluctuation is determined by matching the solution to one in the SOL at the separatrix, the magnetic shear sets the mode radial structure. The radial width $\Delta_{x}$ of the mode inside the separatrix is given by

$$
\Delta_{x} \sim \rho_{s} \sqrt{\frac{2 L_{s}}{k_{y} \rho_{s} L} \frac{s_{m}}{\omega_{b e}}},
$$

where we have taken the effect of the finite inertia of the electrons to dominate over the ohmic resistance of the bulk plasma.

\section{Conclusions}

It has been found that there is no normal ETG-mode for potential fluctuation across a magnetic separatrix for $\tilde{E}_{x} \neq 0$, except for drift wave solutions. For initial value analysis, the amplitude of the potential fluctuation grows like $t^{-\frac{1}{2}} e^{\gamma_{m} t}$ in time, depends weakly on $x$ outside the separatrix, and decays exponentially in $x$ inside the separatrix. The magnetic shear limits the radial extent of the mode.

Finally, as an example of the possible application of this work, we will estimate the characteristics of the ETG-mode developed in this paper for DIII-D boundary plasma parameters. For $R=2.32 \mathrm{~m}, q(a)=6.5, L_{\mathrm{s}}=1.508 \mathrm{~m}, L=q R=15.08 \mathrm{~m}, T_{e}=$ $100 \mathrm{eV}, L_{T_{e}}=1 \mathrm{~cm}, \Lambda=4$, we find that the maximum growth rate $\gamma_{m} \simeq \omega_{m} \simeq 1 \times 10^{6} / \mathrm{s}$ 
is located at $k_{y m} \rho_{s} \sim 0.1$ with $\Delta_{x} \sim 5 \rho_{s}$ to $10 \rho_{s}$. This estimate for radial extent of the mode is somewhat snaller than the width of the transport barrier of several centimeters observed experimentally.

Thus, the conclusion of this work is that, although the ETG-modes in the SOL seems to have a some effect on edge plasma fluctuations, a complete evaluation of the possible relevance of this mode to tokamak plasmas with limiter or divertor awaits the development of nonlinear theory and simulation. Both of these developments are currently under way.

\section{Acknowledgments}

The encouragement and support of Prof. C. K. Birdsall on this problem is gratefully acknowledged. The authors would like to thank Drs. R. H. Cohen and D. L. Pearlstein for helpful comments on normal mode analysis. Our thanks also go to Prof. H. L. Berk, Prof. T. K. Fowler, Dr. W. B. Kunkel, and Dr. D. Hua for useful discussions and to Dr. R. J. Groebner and Dr. R. A. Moyer for providing unpublished edge DIII-D parameters.

This work was supported by the U. S. Department of Energy under contract No. DE-FG03-90ER-54079 with the University of California, Berkeley and contract No. DE-FG03-88ER-53275 with University of California, San Diego. This research was also supported in part by U.S. Office of Neval Research under contract No. N0001489-J-1097 with the University of California, Berkeley. 


\section{Appendix: Normal mode analysis in the SOL}

In the main text, we have demonstrated that the normal mode does not exist when only the transmitted wave through the separatrix is considered in the SOL. In the following, we show that the normal mode exists when a finite length system is considered with an imposed boundary condition $\left.\Phi_{S}(s)\right|_{x=L_{x}}=0$; therefore a reflected wave is included in the analysis.

Starting from Eq. (28) in the main text by replacing $e^{i k_{x, l} x}$ with $\cos \left(k_{x, l} x+\xi\right)$, we have

$$
\Phi_{S}(s)=\sum_{l} C_{l}^{S} \cos \left[k_{\| S, l}(z-L / 2)\right] \cos \left(k_{x, l} x+\xi\right)
$$

From the boundary condition $\left.\Phi_{S}(s)\right|_{x=L_{z}}=0$, we obtain

$$
\begin{aligned}
\cos \left(k_{x, l} L_{x}+\xi\right) & =0 \\
\xi+k_{x, l} L_{x} & =(2 l+1) \frac{\pi}{2},
\end{aligned}
$$

with $l=0, \pm 1, \pm 2, \cdots$. The eigenfunction inside the separatrix is the same as Eq. (32) in the text

$$
\Phi_{E}(s)=\sum_{n} C_{n}^{E} \cos \left[\frac{2 n \pi}{L}(z-L / 2)\right] e^{\alpha_{n} x}
$$

From the matching condition of the radial derivative $\left.\frac{\partial \Phi(s)}{\partial x}\right|_{x=0}$ and $\left.\Phi(s)\right|_{x=0}$ at the separatrix, we have

$$
\begin{aligned}
\sum_{n} C_{n}^{E} \cos \left[\frac{2 n \pi}{L}(z-L / 2)\right] \alpha_{n} & =\sum_{l} C_{l}^{S} \cos \left[k_{\| S, l}(z-L / 2)\right]\left(-k_{x, l} \sin \xi\right)+\frac{\phi(0)}{s+i \omega_{E}-i \omega_{m p_{i}}} \\
\sum_{n} C_{n}^{E} \cos \left[\frac{2 n \pi}{L}(z-L / 2)\right] & =\sum_{l} C_{l}^{S} \cos \left[k_{\| S, l}(z-L / 2)\right] \cos \xi .
\end{aligned}
$$

Multiplying equation (A. 3) by $\cos \left[\frac{2 n \pi}{L}(z-L / 2)\right]$, integrating over $z$ from 0 to $L$, and combining two equations in Eq. (A.3), we obtain an equation similar to Eq. (36):

$$
\sum_{l} \frac{2 C_{l}^{S}}{L} \frac{(-1)^{n} k_{\| S, l} \sin \left(k_{\| S, l} L / 2\right)}{\left[k_{\| S, l}^{2}-\left(\frac{2 \pi n}{L}\right)^{2}\right]}\left(\alpha_{n} \cos \xi-k_{x, l} \sin \xi\right)=\frac{\phi(0) L \delta_{n 0}}{s+i \omega_{E}-i \omega_{* p_{i}}}
$$


For the normal mode $\phi(0)=0$; thus, the physical solution requires the determinant of the matrix to be zero,

$$
\alpha_{n} \cos \xi-k_{x, l} \sin \xi=0
$$

Combining with Eq. (A. 2), we obtain a equation to determine the $k_{x, l}$

$$
\frac{\alpha_{\mathfrak{n}}}{k_{x, l}}=-\cot \left(k_{x, l} L_{x}\right) .
$$

For $\alpha_{n} L_{x}>>1$, we have

$$
k_{x, l}=\frac{l \pi}{2 L_{x}}, l=0, \pm 1, \pm 2, \cdots
$$

Thus the normal ETG-mode exists with the dispersion relation given in Eqs. (30) and (31), and $k_{x, l}$ in Eq. (A. 7). The results for the edge potential fluctuation $\Phi_{E}(\mathbf{x}, t)$ are the same as Eqs. (44) and (51) in the text, apart from a negative square root of the time. 


\section{References}

[1] S. J. Zweben, P. C. Liewer, and R. W. Gould, J. Nucl. Mater. 39, 111 (1982).

[2] Ch. P. Ritz, R. D. Bengtson, S. J. Levinson, and E. J. Powers, Phys. Fluids 27, 2956 (1984).

[3] R. A. Moyer, J. G. Watkins, R. W. Conn, R. Doerner, D. N Hill, R. Lehmer, R. T. McGrath, L. Schmitz, R. D. Stambaugh, and G. Tynan, submitted to J. Nucl. Mater..

[4] J. G. Watkins, R. A. Moyer, D. N. Hill, D. Buchenauer, T. N. Carlstrom, R. W. Conn, J. Cuthbertson, R. Doerner, R. Lehmer, M. A. Mahdavi, R. T. McGrath, L. Schmitz, and R. D. Stambaugh, submitted to J. Nucl. Mater..

[5] K. H. Burrell, T. N. Carlstrom, E. J. Doyle, P. Gohil, R. J. Grrebner, T. Leheclia, N. C. Luhmann, Jr., H. Matsumoto, T. H. Osborne, W. A. Peebles, and R. Philipona, Phys. Fluids B2, 1405 (1990).

[6] W. B. Kunkel and J. U. Guillory, in Proc. 7th Conf. on Phenomena in Ionized Gases, (Belgrade, 1965, Ed. B. Perovic and D. Tocsic) Grad Jevinska Kniga, Belgrade, Yugoslavia(1966), V. 2, 702.

[7] A. V. Nedospasov, Fiz. Plazmy 15 (1989) 1139 [Sov. J. Plasma Phs. 15 ( 1989) 659].

[8] H. L. Berk, D. D. Ryutov and Yu. A. Tsidulko, Phys. Fluids B3, 1346 (1991).

[9] X. Garbet, L. Laurent, J. .P. Roubin, A. Samain, Nucl. Fusion 31, 967 (1991).

[10] H. L. Berk, R. H. Cohen, D. D. Ryutov, Yu. A. Tsidulko and X. Q. Xu, accepted by $\mathrm{Nucl}$. Fusion. 
[11] B. B. Kadomtsev and O. P. Pogutse, "Turbulence in Toroidal System," in Reviews of Plasma Physics, edit by M. A. Leontovich(Consultants Bureau, New York, 1970), Vol. 5, p.249.

[12] H. P. Furth, J. Killeen, and M. N. Rosenbluth, Phys. Fluids 6, 459 (1963).

[13] S. I. Braginskii, in Reviews of Plasma Physics, edit by M. A. Leontovich(Consultants Bureau, New York, 1965), Vol. I, p.205.

[14] H. Capes, Ph. Ghendrih, and A. Samian, Phys. Fluids B4, 1287 (1992).

[15] K. M. Case, Phys. Fluids 3, 143 (1960).

[16] R. A. Smith and M. N. Rosenbluth, Phys. Rev. Lett. 64, 649 (1990).

[17] R. Chodura, Phys. Fluids 25, 1628 (1982).

[18] K. V. Roberts and J. B. Taylor, Phys. Fluids 8, 315 (1965).

[19] M. Gaster, J. Fluid Mech 121, 365 (1982).

[20] Milton Abramowitz and Irene A. Stegun, Handbook of Mathematical Functions, (Dover Publications, Inc., New York, 1972). 


\section{Figures}

Figure 1: Slab model for the boundary plasmas extending across a magnetic separatrix.

Figure 2: A complex $\hat{s}$-plane of Eq. (31) for the $l=0$ mode and $\omega_{* T i}=0$, where $\hat{s}=\left(s+i \omega_{E}\right)\left[\left(\frac{2 \Lambda c_{e}}{L_{T_{e}}}\right)^{2} \nu_{l}\right]^{-\frac{1}{s}}$ and $\hat{k}=k_{y} \rho_{s}\left(\frac{2 \Lambda c_{e}}{L_{T_{e}}}\right)^{-\frac{1}{s}}$. 


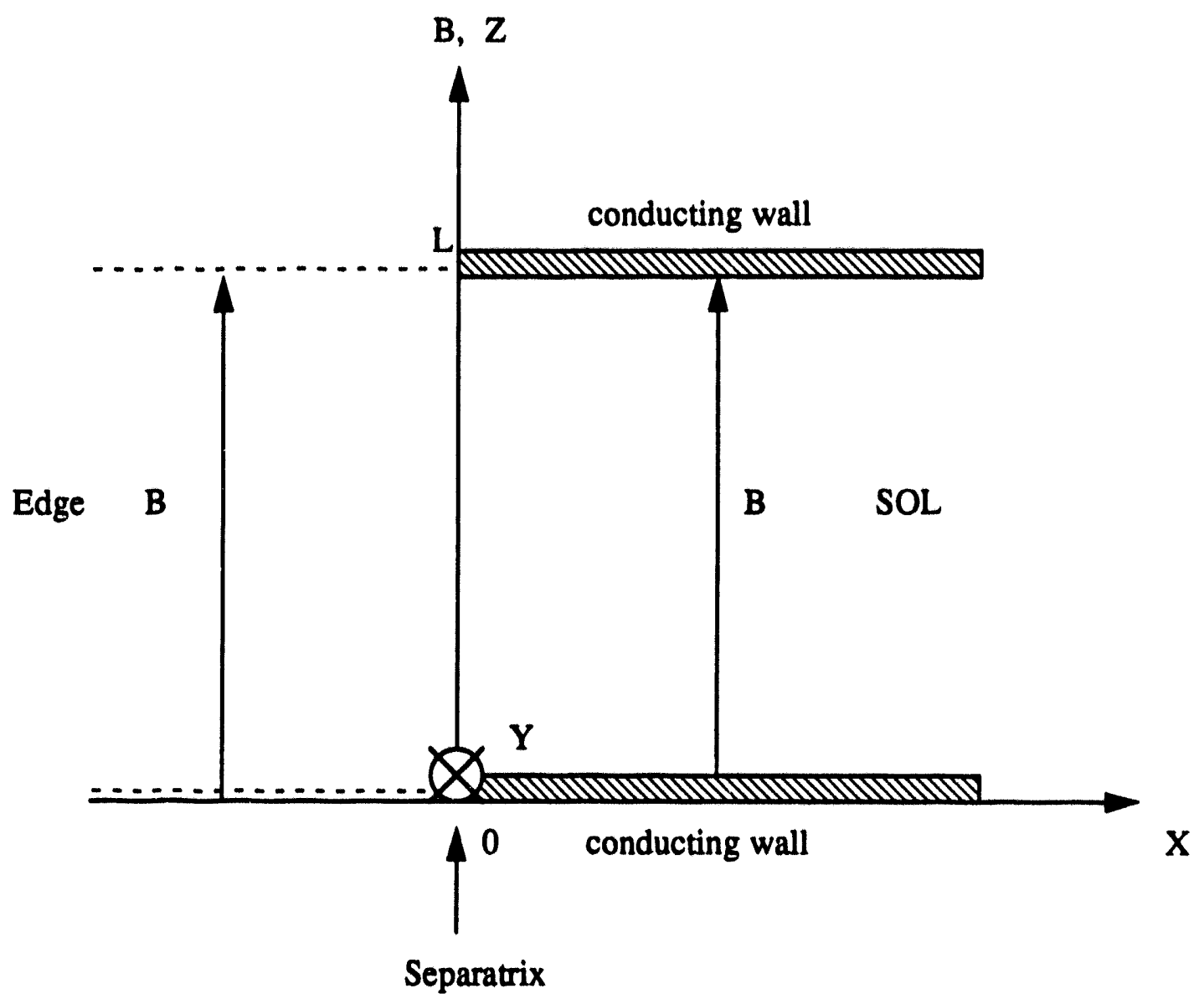




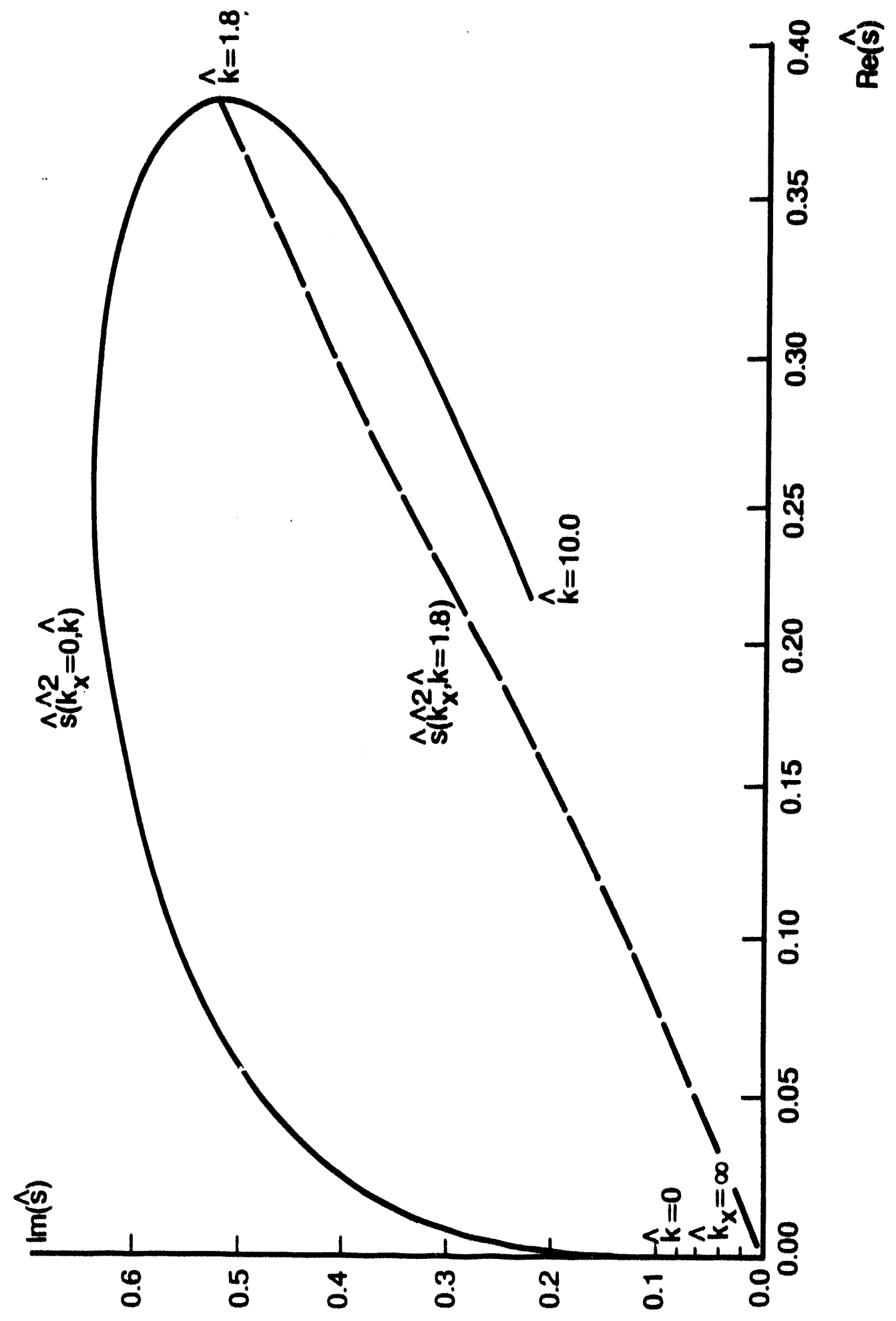



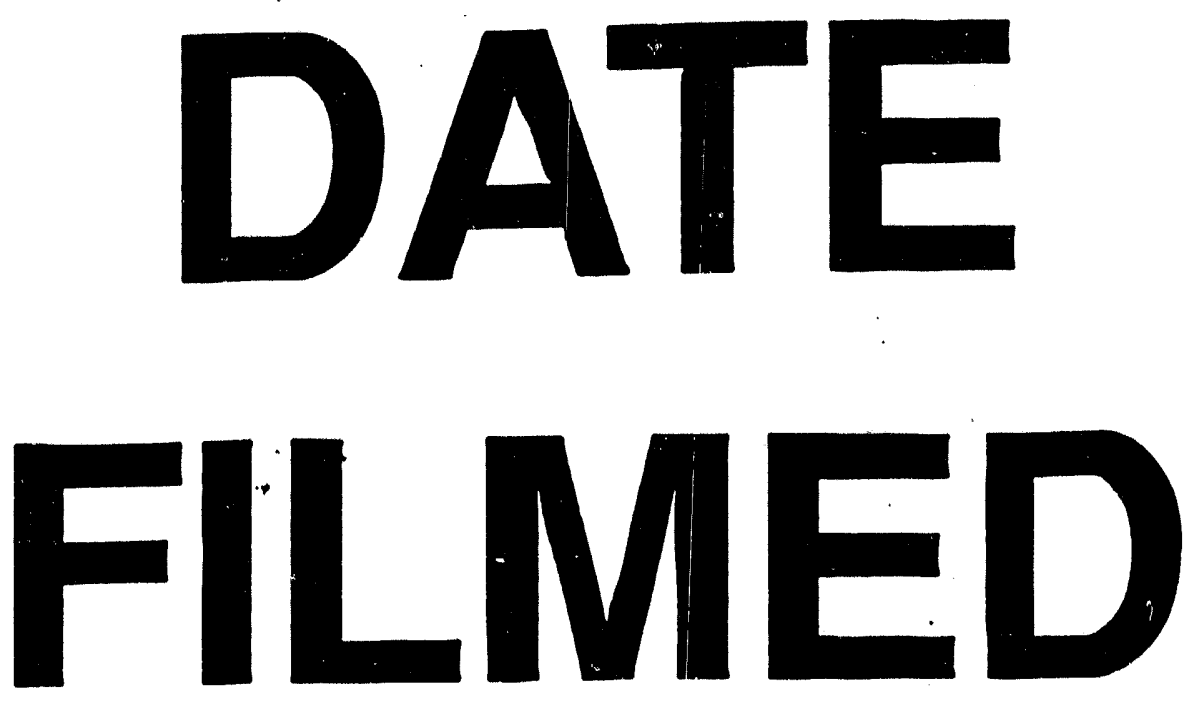

$12 / 6 / 93$
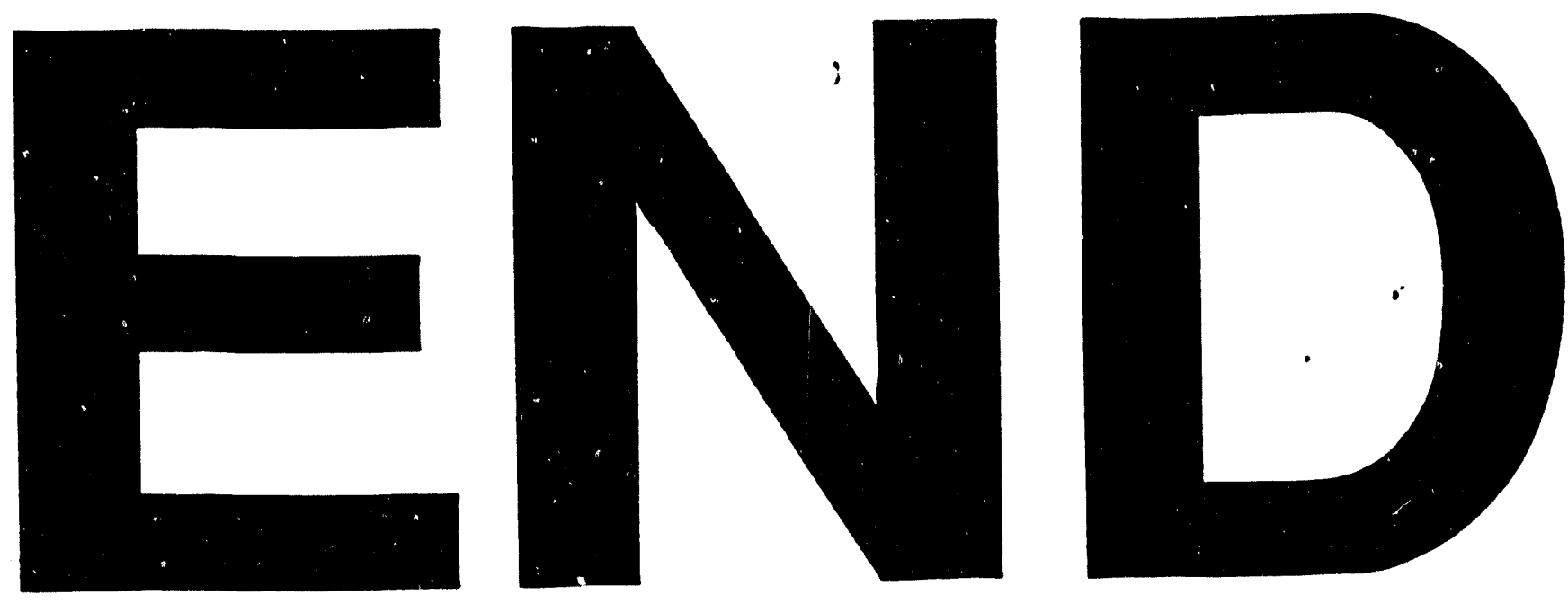
